\title{
The Toxicologist as Educator: Addressing Pain Management in the Midst of an Opioid Epidemic
}

\author{
Gillian A. Beauchamp ${ }^{1}$
}

Published online: 30 June 2015

(C) American College of Medical Toxicology 2015

We call it the "monthly rant" at the Oregon and Alaska Poison Center: a rotating student or resident presents a case of an opioid poisoning and a well-prepared overview of the toxicokinetics and toxicodynamics of opioids in overdose. The eyes of the fellows and attendings meet briefly, and the moment is perfect for an in-depth discussion about the complexities of opioid prescribing, pain management, and pitfalls of opioid therapy. What ensues is a carefully designed discussion surrounding several key components of appropriate pain management and iatrogenic addiction. With an increasing focus on the opioid epidemic and prevention of addiction, medical educators are actively working to design pain management curricula. It is worth highlighting that many toxicologists already incorporate this education for rotators on the toxicology service and that toxicologists are a valuable resource for medical educators involved in the re-designing of medical curricula.

The toxicology rotation seems an ideal place to formally educate trainees to manage pain while attenuating diversion, adverse effects, polypharmacy, and iatrogenic addiction. Whether students and residents spend a day, a week, or a month on the toxicology service, educators can use this opportunity to introduce new approaches toward opioid use in pain management, thus laying the groundwork to reduce morbidity and mortality from opioids. Participants in the discussions on our service typically include third or fourth year medical students, pharmacy students, and emergency

Gillian A. Beauchamp

beauchamp.gillian@gmail.com

1 Department of Emergency Medicine and the Oregon Poison Center, Oregon Health \& Science University, Campus Services Building 559, 3181 SW Sam Jackson Park Road, Portland, OR 97239, USA medicine residents on their toxicology rotation, who will go on to treat patients with acute and chronic painful conditions in all fields of medicine. Even early in training, students and residents make astute observations about the challenges and pitfalls surrounding the management of acute and chronic pain.

Promoting awareness and introducing a primer for good prescribing habits early in training may have a significant impact on the future practices of rotators. One way to do this is to review four key aspects for providing care to the patient with an acute or chronically painful condition. The simplest approach is to integrate this review during toxicology rounds where trainees already participate in opioid exposure and overdose case discussions. The use of a mnemonic such as "RIPE" (review, interview, prescribe appropriately, and educate) provides a guide for trainees, which they can use to approach any patient with acute or chronic pain. Review: teach the trainee to review the patient's chart and, if available, a prescription drug monitoring program. Reviewing a patient's co-morbidities and medications makes it possible to further discuss these issues with the patient during the encounter. Interview: remind the trainee that the history and physical exam include an in-person medication reconciliation to assess for any medication interactions among currently prescribed medications or with medications prescribed during the encounter. Trainees should ask patients about addiction risk factors such as history of psychiatric illness or substance abuse and what social support they have. Prescribe appropriately: advise the trainee to consider when opioid medications are appropriate for use - for emergency medicine residents or emergency medicine-bound students, a recommendation to review resources such as the American College of Emergency Physicians Opioid Guidelines or the Substance Abuse and Mental Health Services Administration Opioid Overdose Toolkit can be helpful $[1,2]$. There are similar guidelines for 
other specialties regarding opioid use [3]. Incorporate a discussion on the studies revealing an increased risk of long-term opioid use after administration in the acute setting [4-6]. Educators can also provide literature introducing the concept of "iatrogenic addiction" [7-11]. Education: remind the trainee that part of treating pain properly involves patient education, including explaining to patients why particular medications are selected for them, the risks and side effects of the medication, and resources available to patients who become dependent on a prescribed medication [12].

As the opioid epidemic continues to gain attention in the medical world and mainstream press, medical educators are moving to standardize the education of providers in appropriate pain management, safer prescribing patterns, and the use of prescription monitoring databases [7-10,13-17]. The toxicology rotation holds great potential as a transformative experience for the medical trainee on this topic, and toxicologists are uniquely poised to seamlessly integrate this training into daily didactics and case discussions. Further, medical toxicologists have the expertise needed to fill the gap in medical education that stems from a lack of formal education in pain management, management of substance use disorders, and the prevention of iatrogenic addiction [18]. The next challenge facing the toxicology community is to leverage our expertise beyond the toxicology rotation by partnering with stakeholders in medical education to re-define formal medical training.

Acknowledgments Special thanks to Dr. Christine Murphy for her assistance in writing this editorial.

Conflict of Interest The author declares no competing interests.

\section{References}

1. American College of Emergency Physicians Opioid Guideline Writing Panel. Clinical policy: critical issues in the prescribing of opioids for adult patients in the emergency department. Ann Emerg Med. 2012;60(4):499-525.

2. Substance Abuse and Mental Health Services Administration Opioid Overdose Toolkit. 2015. Available at http://www.acep.org/ uploadedFiles/ACEP/Clinical_and_Practice_Management/ innovatED/Patient_Treatment/Kaleo $\% 20-\% 20$ SAMHSA $\%$ 20OPIOID\%20OVERDOSE\%20TOOLKIT\%5B3\%5D.pdf. Accessed May 19, 2015.
3. Federation of State Medical Boards. 2013. Model policy for the use of opioid analgesics for the treatment of chronic pain. Washington DC: The Federation. Available at http://www.fsmb.org/Media/ Default/PDF/FSMB/Advocacy/pain_policy_july2013.pdf. Accessed May 31, 2015.

4. Hoppe JA, Kim H, Heard K. Association of emergency department opioid administration with recurrent opioid use. Ann Emerg Med. 2015. doi:10.1016/j.annemergmed.2014.11.015.

5. Alam A, Gomes T, Zheng H, Mamdani MM, Juurlink DN, Bell $\mathrm{CM}$. Long-term analgesic use after low-risk surgery: a retrospective cohort study. Arch Intern Med. 2012;172(5):425-30.

6. Carroll I, Barelka P, Wang CK, Wang BM, Gillespie MJ, McCue R, et al. A pilot cohort study of the determinants of longitudinal opioid use after surgery. Anesth Analg. 2012;115(3):694-702.

7. Beauchamp GA, Winstanley E, Ryan S, Lyons M. Moving beyond misuse and diversion: the urgent need to consider the role of iatrogenic addiction in the current opioid epidemic. Am J Public Health. 2015;104(11):2023-9.

8. Beaudoin FL, Merchant RC, Janicki A, McKaig DM, Babu KM. Preventing iatrogenic overdose: a review of in-emergency department opioid-related adverse drug events and medication errors. Ann Emerg Med. 2015;65(4):423-31.

9. Poon SJ, Greenwood-Ericksen MB. The opioid prescription epidemic and the role of emergency medicine. Ann Emerg Med. 2014;64(5):490-5.

10. Dasgupta N, Kramer ED, Zalman MA, Carino Jr S, Smith MY, Haddox JD, et al. Association between non-medical and prescriptive usage of opioids. Drug Alcohol Depend. 2006;82(2):135-42.

11. Wasan AD, Correll DJ, Kissin I, O'Shea S, Jamison RN. Iatrogenic addiction in patients treated for acute or subacute pain: a systematic review. J Opioid Manag. 2006;2(1):16-22.

12. Substance Abuse and Mental Health Services Administration National Helpline. http://www.samhsa.gov/find-help/nationalhelpline. Accessed May 19, 2015.

13. Paulozzi LJ. Prescription drug overdoses. A review. J Safety Res. 2013;43(4):283-9.

14. Jones CM, Mack KA, Paulozzi LJ. Pharmaceutical overdose deaths, United States 2010. JAMA. 2013;309(7):657-9.

15. McCarthy DM, Wolf MS, McConnell R, Sears J, Chevrier A, Ahlstrom E, et al. Improving patient knowledge and safe use of opioids: a randomized controlled trial. Acad Emerg Med. 2015;22(3):331-9.

16. Logan J, Liu Y, Paulozzi L, Zhang K, Jones C. Opioid prescribing in emergency departments: the prevalence of potentially inappropriate prescribing and misuse. Med Care. 2013;51(8):46-53.

17. Fox TR, Li J, Stevens S, Tippie T. A performance improvement prescribing guideline reduces opioid prescriptions for emergency department dental pain patients. Ann Emerg Med. 2013;62(3): 237-40.

18. Watt-Watson J, McGillion M, Hunter J, Choiniere M, Clark AJ, Dewar A, et al. A survey of prelicensure pain curricula in health science faculties in Canadian universities. Pain Res Manag. 2009;14(6):439-44. 